\title{
Existence and uniqueness of weak solutions for a class of fractional superdiffusion equations
}

\author{
Meilan Qiu', Liquan Mei ${ }^{1 *}$ and Ganshang Yang ${ }^{2,3^{*}}$
}

*Correspondence:
lqmei@mail.xjtu.edu.cn;
ganshanyang@aliyun.com
${ }^{1}$ School of Mathematics and
Statistics, Xi'an Jiaotong University,
Xi'an, 710049, China
2Department of Mathematics,
Yunnan Nationalities University,
Kunming, 650031, China
Full list of author information is
available at the end of the article

"Correspondence:

lqmei@mail.xjtu.edu.cn

anshanyang@aliyun.com

Xi'an, 710049, China

${ }^{2}$ Department of Mathematics,

Yunnan Nationalities University,

Full list of author information is

\begin{abstract}
In this paper, we consider the existence and uniqueness of weak solutions for a class of fractional superdiffusion equations with initial-boundary conditions. For a multidimensional fractional drift superdiffusion equation, we just consider the simplest case with divergence-free drift velocity $u \in L^{2}(\Omega)$ only depending on the spatial variable $x$. Finally, exploiting the Schauder fixed point theorem combined with the Arzelà-Ascoli compactness theorem, we obtain the existence and uniqueness of weak solutions in the standard Banach space $C\left([0, T] ; H_{0}^{1}(\Omega)\right)$ for a class of fractional superdiffusion equations.
\end{abstract}

Keywords: fractional (linear or nonlinear) superdiffusion equation; fractional drift superdiffusion equation; Schauder's fixed point theorem; Arzelà-Ascoli compactness theorem

\section{Introduction}

In recent decades, fractional partial differential equations(FPDEs) have been extensively investigated due to their fbroadness of applications in engineering [1-3], physics, and other fields $[4,5]$, for example, quantum mechanics, the fractal theory and the diffusion in porous media, semiconductor and condensate physics, turbulence, power law phenomenon, viscoelastic system [6-8], biological mathematical and statistical mechanics, the transport of passive tracers carried by fluid flow in a porous medium [9], control and robot, and so on. As is well known, many materials and processes with memory or hereditary properties and nonclassical phenomena in engineering can be described by fractional calculus $[10,11]$. However, since the fractional derivative (or integral) operators are quasidifferential operators, they possess local and the weak singularity properties and usually do not satisfy the semigroup properties, commutative law, and so on, which brought considerable difficulties for theoretical analysis of the fractional partial differential equations. Therefore, it is very important, theoretically and practically, to study such analytic properties of solutions of equations.

To date, most solution techniques for fractional differential equations have exploited the properties of the Fourier and Laplace transforms to confirm a classical solution. For example, in [12], the fundamental solution (or Green function solution) for a time-space fractional diffusion equation with respect to its scaling and similarity properties is inves-

(c) The Author(s) 2017. This article is distributed under the terms of the Creative Commons Attribution 4.0 International License (http://creativecommons.org/licenses/by/4.0/), which permits unrestricted use, distribution, and reproduction in any medium, provided you give appropriate credit to the original author(s) and the source, provide a link to the Creative Commons license, and indicate if changes were made. 
tigated, starting from its Fourier-Laplace representation. Liu et al. [13] considered a time fractional advection dispersion equation and derived the corresponding complete solution. Gorenflo et al. [14] used the Laplace transform method to obtain the scale-invariant solution represented by the Wright function of the time fractional diffusion-wave equation. Through defining suitable functional spaces and their corresponding norms, the time-space fractional diffusion problems can be converted into a weak elliptic problem. Then, by using the existing theory of elliptic problems, a theoretical framework of the variational solutions for the time-space fractional diffusion equation is developed, and the existence and uniqueness of weak solutions are proved in [15]. By the well-known Lax-Milgram theorem, Zhao and Xiao [16] obtained a weak formulation of the multiterm time-space Riesz fractional advection-diffusion equation and proved the existence and uniqueness of weak solutions in the space $B^{\alpha / 2, \beta / 2}(I \times \Omega)(0<\alpha<1,1<\beta<2)$. In addition, many researchers have investigated the initial or boundary value problems of fractional partial differential equations $[17,18]$. Introducing a direct sum Hilbert space, Baleanu and Uğurlu [19] constructed a regular dissipative fractional operator associated with a fractional boundary value problem.

Fixed point theory is an important component of nonlinear functional analysis theory. It is one of the important tools to build the existence and uniqueness of solutions for various equations, including ordinary differential equations, partial differential equations, functional differential equations, and so on. For example, by using the Banach and Schauder fixed point theorems, Chen et al. [20] studied the existence of positive solutions for antiperiodic boundary value problems of nonlinear fractional differential equations. By using the Leggett-Williams fixed point theorem on a convex cone, multiplicity results of positive solutions for the three-point boundary value problems of nonlinear fractional differential equations are obtained in [21]. The contraction mapping principle and the Krasnoselskii fixed point theorem are applied to establish the existence of solutions for an antiperiodic boundary value problem of fractional differential equations of fractional order $q \in(2,3]$ in [22]. Using the fixed point theorem on cones, Bai [23] and Zhang [24] obtained the existence of positive solutions of the Dirichlet-type boundary value problems for nonlinear ordinary differential equations with fractional derivatives. Moreover, there exist many results on the existence and multiplicity of solutions (or positive solutions) of initial value problems for nonlinear fractional differential equations [25]. Using the ArzelàAscoli compact theorem, Qiu et al. [26, 27] focused themselves on the existence of weak (or positive) solutions for the time fractional $p$-Laplace problems in a weighted Sobolev space $H_{0}^{1}(a(x), \Omega)(a(x)$ is a weighted function).

In this paper, motivated by previous research on the existence of weak solutions (or positive solutions) of fractional-order differential equations, our main aim is to develop the Schauder fixed point theorem and the Arzelà-Ascoli compactness theorem for solving a class of time-space fractional initial-boundary value problems with superdiffusion terms. The existence and uniqueness of weak solutions of these equations in a standard Banach space $C\left([0, T] ; H_{0}^{1}(\Omega)\right)$ are obtained. To the best of our knowledge, the results in this paper are new and original since we have not found any discussion in the existing literature.

We mainly consider the following three types of fractional superdiffusion equations. 
1. The time-space fractional superdiffusion equation:

$$
\begin{cases}D^{\alpha} u(x, t)=\epsilon\left(-(-\triangle)^{\frac{\beta}{2}}\right) u(x, t) & \text { in } \Omega_{T}, \\ u(x, t)=0 & \text { on } \partial \Omega_{T}, \\ u(x, 0)=\phi(x) & \text { in } \Omega, \\ u_{t}(x, 0)=\psi(x) & \text { in } \Omega,\end{cases}
$$

where $u \in H^{1}(\Omega)$ is a smooth unknown function, $\Omega_{T}=\Omega \times[0, T], \Omega$ is a bounded region with smooth boundary in $\mathbf{R}^{N}(N \geq 1), D^{\alpha}$ denotes the Caputo fractional derivative of order $\alpha(1<\alpha<2)$ [10], $1<\beta \leq 2$ is the parameter describing the order of the space fractional derivatives, $\epsilon$ is a real positive parameter, and $\phi(x), \psi(x) \in H_{0}^{1}(\Omega)$ are given real-valued smooth functions. The space fractional term in equation (1.1) is defined through the fractional Laplacian operator $\left(-(-\triangle)^{\frac{\beta}{2}}\right) u(x, t)$, which can be thought of as equivalent to the Riesz fractional derivative $\frac{\partial^{\beta}}{\partial|x|^{\beta}} u(x, t)$ under the assumption that $u(x, t), u^{\prime}(x, t), \ldots, u^{(n-1)}(x, t)$ vanish at the end point of an infinite domain (i.e., $x=\mp \infty$ ) $[28,29]$. The Riesz fractional derivative can explain the spatial and temporal nonlocality on finite porous media [30].

2. The time-space fractional nonlinear superdiffusion equation:

$$
\begin{cases}D^{\alpha} u(x, t)+\frac{\partial}{\partial x} f(u)=\epsilon\left(-(-\triangle)^{\frac{\beta}{2}}\right) u(x, t) & \text { in } \Omega_{T}, \\ u(x, t)=0 & \text { on } \partial \Omega_{T}, \\ u(x, 0)=\phi(x) & \text { in } \Omega, \\ u_{t}(x, 0)=\psi(x) & \text { in } \Omega .\end{cases}
$$

All the functions and constants in this equation have the same meaning as those in equation (1.1). In addition, the nonlinear term $f(u)$ is assumed to be Lipschitz continuous on a bounded domain $\Omega$.

The spatial fractional differential operator in equation (1.1) or (1.2) is also an important tool to describe the anomalous diffusion phenomenon; when $1<\beta<2$, it represents a Lévy $\beta / 2$-stable flight [31], and when $\beta \rightarrow 2$, it models a Brownian diffusion process.

3. The multidimensional fractional drift superdiffusion equation:

$$
\begin{cases}D^{\alpha} \theta+\operatorname{div}(u \cdot \theta)=\epsilon \Delta \theta-(-\Delta)^{\gamma} \theta & \text { in } \Omega_{T}, \\ \theta(x, t)=0 & \text { on } \partial \Omega_{T}, \\ \theta(x, 0)=\phi(x) & \text { in } \Omega, \\ \theta_{t}(x, 0)=\psi(x) & \text { in } \Omega,\end{cases}
$$

where $\theta \in H^{1}(\Omega)$ are the smooth unknown vector functions, $\Omega_{T}=\Omega \times[0, T], \Omega$ is a bounded region with smooth boundary conditions in $\mathbf{R}^{N}(N \geq 1), D^{\alpha}(1<\alpha<2)$ denotes the Caputo fractional derivative [10], $\epsilon \Delta \theta(\epsilon>0)$ is an artificial diffusion term [32], and $\phi(x), \psi(x) \in H_{0}^{1}(\Omega)$ are given real-valued functions, the divergence-free drift $u$ is a bounded smooth vector function, and the space fractional term in equation (1.3) is also defined through the fractional Laplacian operator $\left(-(-\Delta)^{\gamma}\right) \theta(x, t), 0<\gamma \leq 1$. In this paper, we only consider the simplest case of the fractional drift superdiffusion equation, that is, the drift velocity $u \in L^{2}(\Omega)$ is time-independent. 
Equations (1.1)-(1.3) have been frequently employed in real-world applications. For their physical meaning and specific applications, we refer to [30,33] and the references therein for more details.

The rest parts of this paper are arranged as follows. In the next section, we first describe the preliminaries of the fractional calculation and then introduce the necessary functional spaces and their corresponding properties. In Section 3, we demonstrate the existence and uniqueness of the weak solutions for a class of fractional superdiffusion models by using the well-known Schauder fixed point theorem and the Arzelà-Ascoli compactness theorem. Finally, we give some concluding remarks in Section 4.

\section{Definitions and some auxiliary results}

For convenience of the reader, we list some basic notations, definitions, and lemmas, which will be used in the subsequent sections. First, we recall the definitions of the RiemannLiouville fractional integrals and fractional derivatives on am interval (finite or infinite) and present some their properties.

Definition 2.1 ([10]) The Riemann-Liouville fractional integral of order $\alpha$ of a function $f(t), t>0$, is defined as

$$
I_{0^{+}}^{\alpha} f(t)=\frac{1}{\Gamma(\alpha)} \int_{0}^{t}(t-s)^{\alpha-1} f(s) d s
$$

provided that the right side is pointwise defined on $(0, \infty)$.

Definition $2.2([10,11])$ The left and right Riemann-Liouville fractional derivatives of order $\alpha(n-1<\alpha<n)$ in an interval $(a, b)(a, b$ can be finite or infinite) can be derived by the Riemann-Liouville fractional integrals as follows:

$$
\begin{array}{ll}
{ }_{a}^{R} D_{x}^{\alpha} u(x)=\frac{1}{\Gamma(n-\alpha)} \frac{d^{n}}{d x^{n}} \int_{a}^{x}(x-\xi)^{n-\alpha-1} u(\xi) d \xi, & x>a, \\
{ }_{x}^{R} D_{b}^{\alpha} u(x)=\frac{(-1)^{n}}{\Gamma(n-\alpha)} \frac{d^{n}}{d x^{n}} \int_{x}^{b}(\xi-x)^{n-\alpha-1} u(\xi) d \xi, & x<b,
\end{array}
$$

where $\Gamma(\cdot)$ is the gamma function. When $\alpha$ is an integer, $D^{\alpha}=d^{\alpha}=d x^{\alpha}$.

Definition 2.3 ([10]) The Caputo fractional derivative of order $\alpha$ of a function $f(t), t>0$, is defined as

$$
D^{\alpha} f(t)=\frac{1}{\Gamma(1-\{\alpha\})} \int_{0}^{t} \frac{1}{(t-s)^{\{\alpha\}}} f^{([\alpha]+1)} d s,
$$

where $\{\alpha\}$ and $[\alpha]$ denote the fractional and integer parts of a real number $\alpha$, respectively, and $\Gamma(\cdot)$ is the gamma function.

Based on the definitions of the Caputo and Riemann-Liouville fractional differential operators, we obtain an immediate consequence $[15,16]$ : for any real order $\beta>0$, we have the following result. 
Lemma 2.1 The Riemann-Liouville fractional derivative and the Caputo fractional derivative are connected with each other by the following relations:

$$
\begin{aligned}
{ }_{0}^{C} D_{t}^{\beta} f(t) & =\frac{1}{\Gamma(-\beta)} \int_{0}^{t}(t-s)^{-\beta-1}\left(f(s)-T_{n-1}[f ; 0](s)\right) d s \\
& =\frac{1}{\Gamma(-\beta)} \int_{0}^{t}(t-s)^{-\beta-1} f(s) d s-\frac{1}{\Gamma(-\beta)} \int_{0}^{t}(t-s)^{-\beta-1} T_{n-1}[f ; 0](s) d s \\
& ={ }_{0}^{R} D_{t}^{\beta} f(t)-{ }_{0}^{R} D_{t}^{\beta} T_{n-1}[f ; 0](t),
\end{aligned}
$$

where $T_{n-1}[f ; 0](t)$ denotes the Taylor polynomial for $f$ of order $n-1$, centered at 0 ,

$$
T_{n-1}[f ; 0](t)=\sum_{k=0}^{n} \frac{t^{k}}{k !} \frac{d^{k}}{d t^{k}} f(0),
$$

where $f(t) \in C^{n-1}[0, T]$, and $C^{n-1}[0, T]$ denotes the space of $n-1$ times continuously differentiable functions on $[0, T]$.

In particular, when $1<\beta<2$, relations (2.5) and (2.6) take the following forms:

$$
{ }_{0}^{C} D_{t}^{\beta} f(t, x)={ }_{0}^{R} D_{t}^{\beta} f(t, x)-\frac{f(0, x)}{\Gamma(1-\beta)}\left(t^{-\beta}\right)-\frac{f^{\prime}(0, x)}{\Gamma(2-\beta)}\left(t^{1-\beta}\right) .
$$

In order to estimate the fractional Laplacian operator term in our problems, we need the following symmetric fractional derivative definition.

Definition 2.4 ([29]) The fractional Riesz derivative of order $\alpha(n-1 \leq \alpha \leq n)$ on an interval $(a, b)(a, b$ can be finite or infinite) is defined as

$$
\frac{\partial^{\alpha}}{\partial|x|^{\alpha}} u(x, t)=-C_{\alpha}\left({ }_{a}^{R} D_{x}^{\alpha}+{ }_{x}^{R} D_{b}^{\alpha}\right) u(x, t),
$$

where ${ }_{a}^{R} D_{x}^{\alpha},{ }_{x}^{R} D_{b}^{\alpha}$ denote the left and right Riemann-Liouville fractional derivatives of $\alpha$ th order, respectively, and $C_{\alpha}=\frac{1}{2 \cos \left(\frac{\pi \alpha}{2}\right)}, \alpha \neq 1$.

Therefore, when the values of parameters $a, b$ are infinite, the fractional Laplacian operator in problems (1.1), (1.2), and (1.3) can be defined by using the idea of fractional calculus from $[29,34,35]$ :

$$
-(-\triangle)^{\frac{\alpha}{2}} u(x, t)=\frac{\partial^{\alpha}}{\partial|x|^{\alpha}} u(x, t)=-\frac{1}{2 \cos (\pi \alpha / 2)}\left(-\infty D_{x}^{\alpha}+{ }_{x} D_{\infty}^{\alpha}\right) u(x, t),
$$

where ${ }_{-\infty} D_{x}^{\alpha}$ and ${ }_{x} D_{\infty}^{\alpha}$ refer to the left and right Riemann-Liouville fractional derivatives of $\alpha$ th order, respectively. In this paper, we mainly base our developments and analysis on restricting the definition to a bounded domain $\Omega$.

In order to establish the weak formulation of fractional superdiffusion problems, we need to introduce a class of fractional-order spaces from [15], where they are defined by fractional derivatives, and we derive some corresponding properties related to these spaces. Let $C^{\infty}(0, T)$ denote the space of infinitely differentiable functions on $(0, T)$, and 
let $C_{0}^{\infty}(0, T)$ denote the space of infinitely differentiable functions with compact support in $(0, T)$. Let $L^{2}(U)$ be the space of measurable functions with Lebesgue-integrable squares in $U$, where the domain $U=I, \Omega$, or $I \times \Omega$, where $I=[0, T]$ is the time domain, and $\Omega=[0, L]$ is the space domain. The inner product and norm in $L^{2}(U)$ are defined by

$$
\begin{aligned}
& (u, v)_{L^{2}(U)}=\int_{U} u v d_{U}, \quad \forall u \in L^{2}(U), \\
& \|u\|_{L^{2}(U)}=(u, u)_{L^{2}(U)}^{\frac{1}{2}}, \quad \forall u, v \in L^{2}(U) .
\end{aligned}
$$

For any real $\sigma>0$, we define the spaces ${ }^{l} H_{0}^{\sigma}(U)$ and ${ }^{r} H_{0}^{\sigma}(U)$ to be the closures of $C_{0}^{\infty}(U)$ with respect to the norms $\|v\|_{l_{H_{0}^{\sigma}(U)}}$ and $\|v\|_{r_{H_{0}^{\sigma}(U)}}$, respectively, where

$$
\begin{aligned}
& \|v\|_{l_{H_{0}^{\sigma}(U)}}:=\left(\|v\|_{L^{2}(U)}^{2}+|v|_{l_{H_{0}^{\sigma}(U)}}^{2}\right)^{\frac{1}{2}}, \\
& |v|_{l_{H_{0}^{\sigma}(U)}^{2}}^{2}:=\left\|{ }_{0}^{R} D_{t}^{\sigma} v\right\|_{L^{2}(U)}^{2}, \\
& \|v\|_{r_{H_{0}^{\sigma}(U)}}:=\left(\|v\|_{L^{2}(U)}^{2}+|v|_{r_{H_{0}^{\sigma}(U)}}^{2}\right)^{\frac{1}{2}}, \\
& |v|_{r_{H_{0}^{\sigma}(U)}}^{2}:=\left\|{ }_{t}^{R} D_{T^{\sigma}}^{\sigma} v\right\|_{L^{2}(U)}^{2} .
\end{aligned}
$$

We also define the norm in the usual Sobolev space $H_{0}^{\sigma}(U)$ as follows:

$$
\begin{aligned}
\|v\|_{H^{\sigma}(U)} & :=\left(\|v\|_{L^{2}(U)}^{2}+|v|_{H_{0}^{\sigma}(U)}^{2}\right)^{\frac{1}{2}}, \\
|v|_{H_{0}^{\sigma}(U)}^{2}: & :=\left(\frac{\left({ }_{0}^{R} D_{t}^{\sigma} \nu,{ }_{t}^{R} D_{T}^{\sigma} v\right)_{L^{2}(U)}}{\cos (\pi \sigma)}\right) .
\end{aligned}
$$

By references [15] and [16] we know that the spaces ${ }^{l} H_{0}^{\sigma}(U),{ }^{r} H_{0}^{\sigma}(U)$, and $H_{0}^{\sigma}(U)$ coincide and that their seminorms are all equivalent to $|v|_{H_{0}^{\sigma}(U)}$ for all $\sigma>0, \sigma \neq n-1 / 2$.

Now we cite some useful lemmas on some fractional operators on the above spaces, which are recalled from $[10,15,16]$.

\section{Lemma 2.2}

(i) For real $0<\beta<1,0<\delta<1$, if $v(0)=0, x \in(0, L)$, then

$$
{ }_{0}^{R} D_{x}^{\beta+\delta} v(x)=\left({ }_{0}^{R} D_{x}^{\beta}\right)\left({ }_{0}^{R} D_{x}^{\delta}\right) v(x)=\left({ }_{0}^{R} D_{x}^{\delta}\right)\left({ }_{0}^{R} D_{x}^{\beta}\right) v(x), \quad \forall v \in H^{\beta+\delta}(0, L) .
$$

(ii) Let $0<\beta<1$. Then, we have

$$
\left({ }_{0}^{R} D_{x}^{\beta} \omega, v\right)_{L^{2}(0, L)}=\left(\omega,{ }_{x}^{R} D_{L}^{\beta} v\right)_{L^{2}(0, L)}, \quad \forall \omega \in H^{\beta}(0, L), v \in C_{0}^{\infty}(0, L) .
$$

Lemma 2.3 Let $0<\beta<2, \beta \neq 1$. Then, for all $\omega, v \in H_{0}^{\frac{\beta}{2}}(0, L)$, we have

$$
\left({ }_{0}^{R} D_{x}^{\beta} \omega, v\right)_{L^{2}(0, L)}=\left({ }_{0}^{R} D_{x}^{\beta / 2} \omega,{ }_{x}^{R} D_{L}^{\beta / 2} v\right)_{L^{2}(0, L)} .
$$

Lemma 2.4 (Schauder fixed point theorem) Let E be a closed convex nonempty subset of a Banach space $X$, and let $F: E \rightarrow E$ be a continuous mapping such that $F(E)$ is a relatively 
compact subset of $X$. Then $F$ has at least one fixed point in $E$, that is, there exists $u \in E$ such that $F(u)=u$.

By Lemmas 2.2 and 2.3 we can define the following space:

$$
B^{\alpha / 2, \beta / 2}(I \times \Omega)=H^{\alpha / 2}\left(I, L^{2}(\Omega)\right) \cap L^{2}\left(I, H_{0}^{\beta / 2}(\Omega)\right), \quad 1<\alpha<2,1<\beta \leq 2,
$$

equipped with the norm

$$
\|v\|_{B^{\alpha / 2, \beta / 2}}(I \times \Omega):=\left(\|v\|_{H^{\alpha / 2}\left(I, L^{2}(\Omega)\right)}^{2}+\|v\|_{L^{2}\left(I, H_{0}^{\beta / 2}(\Omega)\right)}^{2}\right)^{\frac{1}{2}}, \quad \forall v \in B^{\alpha / 2, \beta / 2}(I \times \Omega),
$$

where

$$
H^{\alpha / 2}\left(I, L^{2}(\Omega)\right):=\left\{v:\|v(\cdot, t)\|_{L^{2}(\Omega)} \in H^{\alpha / 2}(I)\right\}
$$

endowed with the norm

$$
\|v\|_{H^{\alpha / 2}\left(I, L^{2}(\Omega)\right)}:=\|\| v(\cdot, t)\left\|_{L^{2}(\Omega)}\right\|_{H^{\alpha / 2}(I)} .
$$

Observe that $B^{\alpha / 2, \beta / 2}(I \times \Omega)$ is a closed bounded convex subspace (subset) of the standard Banach space $C\left([0, T] ; H_{0}^{1}(\Omega)\right)$. By recalling the same argument as in Theorem 3.1 of [15] we obtain the following:

Theorem 2.5 Assume that $1<\alpha<2,1<\beta \leq 2$, and $u_{t}(x, 0)=0$. Then, system (1.1) has a unique weak solution in the space $B^{\alpha / 2, \beta / 2}(I \times \Omega)$. Furthermore,

$$
\|u\|_{B^{\alpha / 2, \beta / 2(I \times \Omega)}} \leq\|u(x, 0)\|_{L^{2}(\Omega)}\left\|t^{-\alpha}\right\|_{L^{q}(\Omega)} \leq\|\phi(x)\|_{L^{2}(\Omega)}\left\|t^{-\alpha}\right\|_{L^{q}(\Omega)}, \quad q=\frac{2}{\alpha} .
$$

\section{Existence and uniqueness of weak solutions}

In this section, we mainly discuss the existence and uniqueness of weak solutions for three fractional superdiffusion problems (1.1), (1.2), and (1.3) in the standard Banach space $C\left([0, T] ; H_{0}^{1}(\Omega)\right)$. First, we need the following several lemmas.

Lemma 3.1 ([11]) Let $\alpha>0$. Then

$$
I^{\alpha C} D^{\alpha} u(t)=u(t)+c_{0}+c_{1} t+c_{2} t^{2}+\cdots+c_{n-1} t^{n-1}
$$

for some $c_{i} \in R, i=0,1,2, \ldots, n-1, n=[\alpha]+1$.

Lemma 3.2 Let $y \in C[0, T], T>0,1<\alpha<2$. Then the problem

$$
D^{\alpha} u(t)=y(t), \quad t \in[0, T],
$$

has the unique solution

$$
u(t)=u(0)+u^{\prime}(0) t+\frac{1}{\Gamma(\alpha)} \int_{0}^{t}(t-s)^{\alpha-1} y(s) d s
$$


Lemma 3.3 (See [15]) For real $\theta>0$ and $v \in C_{0}^{\infty}(R)$, we have

$$
\begin{aligned}
& \left({ }_{-\infty}^{R} D_{x}^{\theta} v(x),{ }_{x}^{R} D_{\infty}^{\theta} v(x)\right)_{L^{2}(R)}=\cos (\pi \theta)\left\|_{-\infty}^{R} D_{x}^{\theta} v(x)\right\|_{L^{2}(R)}^{2}, \\
& \left({ }_{-\infty}^{R} D_{x}^{\theta} v(x),{ }_{x}^{R} D_{\infty}^{\theta} v(x)\right)_{L^{2}(R)}=\cos (\pi \theta)\left\|_{x}^{R} D_{\infty}^{\theta} v(x)\right\|_{L^{2}(R)}^{2} .
\end{aligned}
$$

Lemma 3.4 ([36], Thm. 4.1.2) Let $s$ and $t$ be real numbers such that $s \geq t$. Then

$$
H^{s}\left(R^{n}\right) \hookrightarrow H^{t}\left(R^{n}\right)
$$

Lemma 3.5 ([37]) Let $\left(H^{s}\left(R^{n}\right)\right)^{\prime}$ denote the dual space of $H^{s}\left(R^{n}\right)$. Then

$$
\left(H^{s}\left(R^{n}\right)\right)^{\prime} \simeq H^{-s}\left(R^{n}\right)
$$

in the sense of algebra and topology, and for all $v \in H^{-s}\left(R^{n}\right)$, if $l \in\left(H^{s}\left(R^{n}\right)\right)^{\prime}$ is a continuous linear functional operator, then we have

$$
\|v\|_{H^{-s}\left(R^{n}\right)}=\|l\|_{\left(H^{s}\left(R^{n}\right)\right)^{\prime}} \leq C,
$$

where $C$ is a positive constant.

\subsection{Existence and uniqueness of weak solutions for the time-space fractional superdiffusion equation}

By Lemma 3.2 problem (1.1) can be reduced to an equivalent integral equation under the fractional-order integral operator $I^{\alpha}$ as in the following problem:

$$
\begin{cases}-\phi(x)-\psi(x) t+u(x, t)=\frac{1}{\Gamma(\alpha)} \int_{0}^{t}(t-s)^{\alpha-1} \epsilon\left(-(-\Delta)^{\beta / 2}\right) u(x, t) d s, & \text { in } \Omega_{T}, \\ u(x, t)=0, & \text { on } \partial \Omega_{T} .\end{cases}
$$

The functional integral equations describe many physical phenomena in various areas of natural science, mathematical physics, mechanics, and population dynamics [38, 39]. The theory of integral equations is developing rapidly with the help of tools in functional analysis, topology, and fixed point theory (see, e.g., [40-42]), and it serves as a useful tool in turn for other branches of mathematics, for example, for differential equations [43]. Now, we define

$$
\begin{cases}\Phi(u)=\phi(x)+\psi(x) t+\frac{1}{\Gamma(\alpha)} \int_{0}^{t}(t-s)^{\alpha-1} \epsilon\left(-(-\Delta)^{\beta / 2}\right) u(x, t) d s, & \text { in } \Omega_{T}, \\ u(x, t)=0, & \text { on } \partial \Omega_{T} .\end{cases}
$$

Definition 3.1 We call $u \in C\left([0, T] ; H_{0}^{1}(\Omega)\right)$ a weak solution of the time-space fractional order superdiffusion equation (1.1) if $\int_{\Omega}(u-\Phi(u)) v d x=0$ for all $t \in[0, T]$ and every $v \in$ $H_{0}^{1}(\Omega)$, that is,

$$
\int_{\Omega} u v d x=\int_{\Omega}\left[\phi(x)+\psi(x) t+\frac{1}{\Gamma(\alpha)} \int_{0}^{t}(t-s)^{\alpha-1} \epsilon\left(-(-\Delta)^{\beta / 2}\right) u(x, s) d s\right] v d x .
$$

We know that $B^{\alpha / 2, \beta / 2}(I \times \Omega) \subset C\left([0, T] ; H_{0}^{1}(\Omega)\right)$ by the definition of (2.15), and combining with the results of Theorem 2.5 , we have the following theorem. 
Theorem 3.6 If $1<\alpha<2,1<\beta \leq 2$, and $u_{t}(x, 0)=0$, then the operator $\Phi(u): B^{\alpha / 2, \beta / 2}(I \times$ $\Omega) \rightarrow B^{\alpha / 2, \beta / 2}(I \times \Omega)$ is completely continuous.

Proof Put $F(u)=\epsilon\left(-(-\Delta)^{\beta / 2}\right) u(x, t)$. Then

$$
\Phi(u)=\phi(x)+\psi(x) t+\frac{1}{\Gamma(\alpha)} \int_{0}^{t}(t-s)^{\alpha-1} F(u) d s, \quad \forall u, v \in B^{\alpha / 2, \beta / 2}(I \times \Omega) .
$$

For each $v \in H_{0}^{1}(\Omega)$, using integration by parts, in terms of the Riesz fractional derivative in Definition 2.4 and Lemma 3.3, since $1<\beta \leq 2$, by Lemma 3.4 together with the realorder Sobolev imbedding theorem, we get that $H_{0}^{1}(\Omega) \hookrightarrow H_{0}^{\beta / 2}(\Omega) \hookrightarrow L^{2}(\Omega)$, and thus, $\|u\|_{L^{2}}(\Omega) \leq\|u\|_{H_{0}^{\beta / 2}(\Omega)} \leq\|u\|_{H_{0}^{1}(\Omega)}$. We further denote $\|u\|_{H_{0}^{1}(\Omega)}$ and $\|u\|_{H_{0}^{-1}(\Omega)}$ by $\|u\|_{H_{0}^{1}}$ and $\|u\|_{H_{0}^{-1}}$ respectively. Hence, by the Cauchy-Schwarz and Hölder inequalites, the standard Sobolev imbedding theorem, Theorem 2.5, and Lemma 3.5, since $1<\alpha<2$, for any $v \in$ $H_{0}^{1}(\Omega)$ satisfying $\|v\|_{H_{0}^{1}(\Omega)} \leq 1$, we obtain

$$
\begin{aligned}
|\langle F(u), v\rangle| & =\left|\int_{\Omega} \epsilon\left(-(-\Delta)^{\beta / 2}\right) u(x, t) v(x, t) d s\right| \\
& =\left|\left(\epsilon\left(-(-\Delta)^{\beta / 2}\right) u(x, t), v(x, t)\right)\right|=\left|\left(\epsilon^{R} D_{|x|}^{\beta} u(x, t), v(x, t)\right)\right| \\
& =\left|-\epsilon C_{\beta}\left[\left({ }_{a}^{R} D_{x}^{\beta / 2} u,{ }_{x}^{R} D_{b}^{\beta / 2} v\right)_{L^{2}(U)}+\left({ }_{x}^{R} D_{b}^{\beta / 2} u,{ }_{a}^{R} D_{x}^{\beta / 2} v\right)_{L^{2}(U)}\right]\right| \\
& \leq \epsilon C_{\beta}\left[\left\|{ }_{a}^{R} D_{x}^{\beta / 2} u\right\|_{L^{2}(U)}\left\|{ }_{x}^{R} D_{b}^{\beta / 2} v\right\|_{L^{2}(U)}+\left\|{ }_{x}^{R} D_{b}^{\beta / 2} u\right\|_{L^{2}(U)}\left\|{ }_{a}^{R} D_{x}^{\beta / 2} v\right\|_{L^{2}(U)}\right] \\
& \leq 2 \epsilon C_{\beta}\|u\|_{L^{2}\left(I, H^{\beta / 2}(\Omega)\right)}\|v\|_{H_{0}^{\alpha / 2}\left(I, L^{2}(\Omega)\right)} \leq 2 \epsilon C_{\beta}\|u\|_{B^{\alpha / 2, \beta / 2}(I \times \Omega)}\|v\|_{H_{0}^{1}(I \times \Omega)} \\
& \leq 2 \epsilon C_{\beta}\|u\|_{B^{\alpha / 2, \beta / 2}(I \times \Omega)}\|v\|_{H_{0}^{1}(\Omega)} \\
& \leq 2 \epsilon C_{\beta}\|\phi(x)\|_{L^{2}(U)}\left\|t^{-\alpha}\right\|_{L^{q}(U)}=M_{1} .
\end{aligned}
$$

Here $C_{\beta}=\frac{1}{2 \cos \left(\frac{\pi \beta}{2}\right)}$, and $q=\frac{2}{\alpha}, M_{1}$ is a positive constant. Thus,

$$
\begin{aligned}
\|\Phi(u)\|_{H^{-1}}= & \sup _{\|v\| H_{0}^{1} \leq 1}|\langle\Phi(u), v\rangle| \\
= & \sup _{\|v\| H_{0}^{1} \leq 1}\left|\langle\phi(x), v\rangle+\langle\psi(x), v\rangle t+\frac{1}{\Gamma(\alpha)} \int_{0}^{t}(t-s)^{\alpha-1}\langle F(u), v\rangle d s\right| \\
\leq & \|\phi(x)\|_{L^{\infty}(\Omega)}\|v\|_{H_{0}^{1}(\Omega)}+\|\psi(x)\|_{L^{\infty}(\Omega)}\|v\|_{H_{0}^{1}(\Omega)} T \\
& +|\langle F(u), v\rangle|\left|\frac{1}{\Gamma(\alpha)} \int_{0}^{t}(t-s)^{\alpha-1} d s\right| \\
\leq & \|\phi(x)\|_{L^{\infty}(\Omega)}\|v\|_{H_{0}^{1}(\Omega)}+\|\psi(x)\|_{L^{\infty}(\Omega)}\|v\|_{H_{0}^{1}(\Omega)} T \\
& +2 \epsilon C_{\beta}\|\phi(x)\|_{L^{2}(\Omega)}\left\|t^{-\alpha}\right\|_{L^{q}(\Omega)} \frac{1}{\Gamma(\alpha)}\left|\int_{0}^{t}(t-s)^{\alpha-1} d s\right| \\
\leq & \|\phi(x)\|_{L^{\infty}(\Omega)}+\|\psi(x)\|_{L^{\infty}(\Omega)} T+\frac{M_{1}}{\alpha \Gamma(\alpha)} t^{\alpha} \\
\leq & \|\phi(x)\|_{L^{\infty}(\Omega)}+\|\psi(x)\|_{L^{\infty}(\Omega)} T+\frac{M_{1}}{\Gamma(\alpha+1)} T^{\alpha} .
\end{aligned}
$$

Hence, $\Phi(u)$ is a bounded operator. 
On the other hand, given $\epsilon>0$, set

$$
\delta=\min \left\{\frac{\epsilon}{4\|\psi(x)\|_{L^{\infty}(\mid \Omega)}}, \frac{1}{2}\left(\frac{\epsilon \Gamma(\alpha)}{2 M_{1}}\right)^{\frac{1}{\alpha}}\right\}
$$

Then, for every $v \in H_{0}^{1}(\Omega)$ and $t_{1}, t_{2} \in[0, T]$ such that $0<t_{2}-t_{1}<\delta$, we have

$$
\left\|\Phi u\left(t_{2}\right)-\Phi u\left(t_{1}\right)\right\|_{H^{-1}}=\sup _{\|v\|_{H_{0}^{1}} \leq 1}\left|\left\langle\Phi u\left(t_{2}\right)-\Phi u\left(t_{1}\right), v\right\rangle\right| \leq \epsilon
$$

that is, $\Phi(u)$ is equicontinuous. In fact,

$$
\begin{aligned}
\| \Phi & u\left(t_{2}\right)-\Phi u\left(t_{1}\right) \|_{H^{-1}} \\
= & \sup _{\|v\|_{H_{0}^{1}} \leq 1}\left|\left\langle\Phi u\left(t_{2}\right)-\Phi u\left(t_{1}\right), v\right\rangle\right| \\
= & \sup _{\|v\|_{H_{0}^{1}} \leq 1} \mid\langle\psi(x), v\rangle\left(t_{2}-t_{1}\right)+\frac{1}{\Gamma(\alpha)} \int_{0}^{t_{2}}\left(t_{2}-s\right)^{\alpha-1}\langle F(u), v\rangle d s \\
& -\frac{1}{\Gamma(\alpha)} \int_{0}^{t_{1}}\left(t_{1}-s\right)^{\alpha-1}\langle F(u), v\rangle d s \mid \\
\leq & \|\psi(x)\|_{L^{\infty}(\Omega)}\|v\|_{H_{0}^{1}}\left|t_{2}-t_{1}\right|+|\langle F(u), v\rangle|\left|\frac{1}{\Gamma(\alpha)} \int_{t_{1}}^{t_{2}}\left(t_{2}-s\right)^{\alpha-1} d s\right| \\
& +|\langle F(u), v\rangle|\left|\frac{1}{\Gamma(\alpha)} \int_{0}^{t_{1}}\left(\left(t_{2}-s\right)^{\alpha-1}-\left(t_{1}-s\right)^{\alpha-1}\right) d s\right| \\
\leq & \|\psi(x)\|_{L^{\infty}(\Omega)}\left|t_{2}-t_{1}\right|+\frac{M_{1}}{\Gamma(\alpha)} \int_{t_{1}}^{t_{2}}\left|\left(t_{2}-s\right)^{\alpha-1}\right| d s \\
\leq & \| \frac{M_{1}}{\Gamma(\alpha)} \int_{0}^{t_{1}}\left|\left(t_{2}-s\right)^{\alpha-1}-\left(t_{1}-s\right)^{\alpha-1}\right| d s \\
\leq & \|\psi(x)\|_{L^{\infty}(\Omega)}\left|t_{2}-t_{1}\right|+\frac{M_{1}}{\alpha \Gamma(\alpha)}\left(t_{2}^{\alpha}-t_{1}^{\alpha}\right) . \\
& =t_{1}-t_{1} \mid+\frac{M_{1}}{\alpha \Gamma(\alpha)} t_{2}^{\alpha}-\frac{M_{1}}{\alpha \Gamma(\alpha)} t_{1}^{\alpha}
\end{aligned}
$$

In the following, we divide the proof into two cases.

Case 1: $\delta \leq t_{1}<t_{2}<T$. Since $1<\alpha<2$, we get

$$
\begin{aligned}
& \left\|\Phi u\left(t_{2}\right)-\Phi u\left(t_{1}\right)\right\|_{H^{-1}} \\
& =\sup _{\|v\|_{H_{0}^{1}} \leq 1}\left|\left\langle\Phi u\left(t_{2}\right)-\Phi u\left(t_{1}\right), v\right\rangle\right| \\
& \leq\|\psi(x)\|_{L^{\infty}(\Omega)}\left|t_{2}-t_{1}\right|+\frac{M_{1}}{\alpha \Gamma(\alpha)}\left(t_{2}^{\alpha}-t_{1}^{\alpha}\right) \\
& \quad=\|\psi(x)\|_{L^{\infty}(\Omega)}\left|t_{2}-t_{1}\right|+\frac{M_{1}}{\alpha \Gamma(\alpha)} \alpha t^{\alpha-1}\left(t_{2}-t_{1}\right) \\
& \leq\|\psi(x)\|_{L^{\infty}(\Omega)}\left|t_{2}-t_{1}\right|+\frac{M_{1}}{\Gamma(\alpha)} \delta^{\alpha}
\end{aligned}
$$




$$
\begin{aligned}
& <\|\psi(x)\|_{L^{\infty}(\Omega)} \delta+\frac{M_{1}}{\Gamma(\alpha)} \delta^{\alpha} \\
& <\frac{1}{2} \times \frac{\epsilon}{2}+\left(\frac{1}{2}\right)^{\alpha} \frac{\epsilon}{2}<\frac{\epsilon}{2}+\frac{\epsilon}{2}=\epsilon .
\end{aligned}
$$

Here $t_{1}<t<t_{2}$, and we applied the mean theorem $t_{2}^{\alpha}-t_{1}^{\alpha}=\alpha t^{\alpha-1}\left(t_{2}-t_{1}\right)$.

Case 2: $0 \leq t_{1}<\delta, t_{2}<2 \delta$. We have

$$
\begin{aligned}
\left\|\Phi u\left(t_{2}\right)-\Phi u\left(t_{1}\right)\right\|_{H^{-1}} & \left.=\sup _{\|v\|_{H_{0}^{1}} \leq 1}|| \Phi u\left(t_{2}\right)-\Phi u\left(t_{1}\right), v\right\rangle \mid \\
& \leq\|\psi(x)\|_{L^{\infty}(\Omega)}\left|t_{2}-t_{1}\right|+\frac{M_{1}}{\alpha \Gamma(\alpha)}\left(t_{2}^{\alpha}-t_{1}^{\alpha}\right) \\
& \leq\|\psi(x)\|_{L^{\infty}(\Omega)} \delta+\frac{M_{1}}{\alpha \Gamma(\alpha)} t_{2}^{\alpha} \\
& \leq\|\psi(x)\|_{L^{\infty}(\Omega)} \delta+\frac{M_{1}}{\Gamma(\alpha+1)}(2 \delta)^{\alpha} \\
& <\frac{\epsilon}{2}+\frac{\epsilon}{2}=\epsilon .
\end{aligned}
$$

Therefore, $\Phi$ is equicontinuous and uniformly bounded. The Arzelà-Ascoli compactness theorem implies that $\Phi$ is compact on $B^{\alpha / 2, \beta / 2}(I \times \Omega)$, and so the operator $\Phi(u)$ : $B^{\alpha / 2, \beta / 2}(I \times \Omega) \rightarrow B^{\alpha / 2, \beta / 2}(I \times \Omega)$ is completely continuous. This completes the proof of the Theorem 3.6.

Since $B^{\alpha / 2, \beta / 2}(I \times \Omega) \subset C\left([0, T] ; H_{0}^{1}(\Omega)\right)$, combining the results of Theorem 3.6 with the Schauder fixed point theorem (Lemma 2.4), we obtain that the time-space fractional superdiffusion problem (1.1) has a unique weak solution $u \in C\left([0, T] ; H_{0}^{1}(\Omega)\right)$.

\subsection{Existence and uniqueness of weak solutions for the time-space fractional nonlinear superdiffusion equation}

In this part, we adopt the same Banach space with its norm and properties and the same ideas as in Section 3.1. We also need the following lemma.

Lemma 3.7 Suppose that there exists a constant $L>0$ such that

$$
|f(u)-f(v)| \leq L|u-v|, \quad \forall t \in I, u, v \in \Omega
$$

Then $f(u)$ is bounded in $\Omega$, and thus, there exists a positive constant $N$ such that

$$
|f(u)| \leq N, \quad \forall t \in I, u \in \Omega
$$

Similarly to Section 3.1, by Definitions 2.1 and 2.3 we have that

$$
I^{\alpha} D^{\alpha} u=\frac{1}{\Gamma(\alpha) \Gamma(1-\{\alpha\})} \int_{0}^{t} \int_{0}^{\tau}(t-\tau)^{\alpha-1}(\tau-s)^{-\{\alpha\}} u^{([\alpha]+1)}(s) d s d \tau
$$


Taking $\tau=(t-s) \lambda+s$, we have

$$
\begin{aligned}
I^{\alpha} D^{\alpha} u & =\frac{1}{\Gamma(\alpha) \Gamma(1-\{\alpha\})} \int_{0}^{t} \int_{0}^{1}(t-s)^{\alpha-\{\alpha\}-1}(1-\lambda)^{-\{\alpha\}} \lambda^{-\{\alpha\}} d_{\lambda} u^{([\alpha]+1)}(s) d s \\
& =\frac{1}{\Gamma(\alpha) \Gamma(1-\{\alpha\})} \int_{0}^{t} B(\alpha, 1-\{\alpha\})(t-s)^{[\alpha]} u^{([\alpha]+1)}(s) d s \\
& =\frac{1}{[\alpha] !} \int_{0}^{t}(t-s)^{[\alpha]} u^{([\alpha]+1)}(s) d s .
\end{aligned}
$$

Integrating by parts repeatedly, we obtain

$$
\begin{aligned}
I^{\alpha} D^{\alpha} u & =-\sum_{k=1}^{[\alpha]} \frac{u^{([\alpha]-k)}(0)}{([\alpha]-k) !} t^{[\alpha]-k}+\int_{0}^{t} u^{\prime}(s) d s, \\
& =-\sum_{k=0}^{[\alpha]} \frac{u^{([\alpha]-k)}(0)}{([\alpha]-k) !} t^{[\alpha]-k}+u(t) .
\end{aligned}
$$

When $\alpha \in(1,2), I^{\alpha} D^{\alpha} u=-u(0)-u^{\prime}(0) t+u(t)$. Combining with Lemma 3.2, we can reduce problem (1.2) to an equivalent integral equation under the fractional-order integral operator $I^{\alpha}$ as in the following problem:

$$
\begin{cases}-\phi(x)-\psi(x) t+u(x, t) & \\ \quad=\frac{1}{\Gamma(\alpha)} \int_{0}^{t}(t-s)^{\alpha-1}\left(-\frac{\partial}{\partial x} f(u)+\epsilon\left(-(-\Delta)^{\beta / 2}\right)\right) u(x, t) d s & \text { in } \Omega_{T}, \\ u(x, t)=0 & \text { on } \partial \Omega_{T} .\end{cases}
$$

Now, we define

$$
\left\{\begin{aligned}
\Phi(u)= & \phi(x)+\psi(x) t & & \\
& +\frac{1}{\Gamma(\alpha)} \int_{0}^{t}(t-s)^{\alpha-1}\left(-\frac{\partial}{\partial x} f(u)+\epsilon\left(-(-\Delta)^{\beta / 2}\right)\right) u(x, s) d s & & \text { in } \Omega_{T} \\
u(x, t)= & & & \text { on } \partial \Omega_{T} .
\end{aligned}\right.
$$

Definition 3.2 We call $u \in C\left([0, T] ; H_{0}^{1}(\Omega)\right)$ a weak solution of the time-space fractionalorder nonlinear superdiffusion equation (1.2) if $\int_{\Omega}(u-\Phi(u)) v d x=0, \forall t \in[0, T]$ for every $v \in H_{0}^{1}(\Omega)$, that is,

$$
\left.\int_{\Omega} u v d x=\int_{\Omega}\left[\phi(x)+\psi(x) t+\frac{1}{\Gamma(\alpha)} \int_{0}^{t}(t-s)^{\alpha-1}\left(\epsilon\left(-(-\Delta)^{\beta / 2}\right)\right) u(x, s)-\frac{\partial f(u)}{\partial x}\right) d s\right] v d x .
$$

By the definition of (2.15) we have $B^{\alpha / 2, \beta / 2}(I \times \Omega) \subset C\left([0, T] ; H_{0}^{1}(\Omega)\right)$, and combining the results of Theorem 2.5 again, we obtain the following theorem.

Theorem 3.8 Let $1<\alpha<2,1<\beta \leq 2$, and $u_{t}(x, 0)=0$. Then the operator $\Phi(u): B^{\alpha / 2, \beta / 2}(I \times$ $\Omega) \rightarrow B^{\alpha / 2, \beta / 2}(I \times \Omega)$ is completely continuous.

Proof Put $F(u)=\epsilon\left(-(-\Delta)^{\beta / 2}\right) u(x, s)+\frac{\partial}{\partial x} f(u)$. We can rewrite

$$
\Phi(u)=\phi(x)+\psi(x) t+\frac{1}{\Gamma(\alpha)} \int_{0}^{t}(t-s)^{\alpha-1} F(u) d s, \quad \forall u \in B^{\alpha / 2, \beta / 2}(I \times \Omega) .
$$


For any $v \in H_{0}^{1}(\Omega)$ satisfying $\|v\|_{H_{0}^{1}(\Omega)} \leq 1$, we have

$$
\begin{aligned}
|\langle F(u), v\rangle|= & \left|\left\langle\epsilon\left(-(-\Delta)^{\beta / 2}\right) u(x, t)+\frac{\partial}{\partial x} f(u), v\right\rangle\right| \\
= & \left|\left\langle\epsilon^{R} D_{|x|}^{\beta} u(x, t)+\frac{\partial}{\partial x} f(u), v\right\rangle\right| \\
\leq & \left|\left\langle-\epsilon C_{\beta}\left({ }_{a}^{R} D_{x}^{\beta}+{ }_{x}^{R} D_{b}^{\beta}\right), v\right\rangle\right|+\left|\left\langle\frac{\partial f(u)}{\partial x}, v\right\rangle\right| \\
\leq & \left|-\epsilon C_{\beta}\left[\left({ }_{a}^{R} D_{x}^{\beta / 2} u,{ }_{x}^{R} D_{b}^{\beta / 2} v\right)_{L^{2}(U)}+\left({ }_{x}^{R} D_{b}^{\beta / 2} u,_{a}^{R} D_{x}^{\beta / 2} v\right)_{L^{2}(U)}\right]\right|+|f(u) \cdot \Delta v| \\
\leq & \epsilon C_{\beta}\left[\left\|{ }_{a}^{R} D_{x}^{\beta / 2} u\right\|_{L^{2}(U)}\left\|{ }_{x}^{R} D_{b}^{\beta / 2} v\right\|_{L^{2}(U)}\right. \\
& \left.+\left\|{ }_{x}^{R} D_{b}^{\beta / 2} u\right\|_{L^{2}(U)}\left\|{ }_{a}^{R} D_{x}^{\beta / 2} v\right\|_{L^{2}(U)}\right]+|f(u)| \cdot\|\Delta v\|_{L^{2}(\Omega)} \\
\leq & \epsilon C_{\beta}\|u\|_{L^{2}\left(I, H^{\beta / 2}(\Omega)\right)}\|v\|_{L^{2}(I \times \Omega)} \\
& +\epsilon C_{\beta}\|u\|_{L^{2}\left(I, H^{\beta / 2}(\Omega)\right)}\|v\|_{L^{2}(I \times \Omega)}+N\|v\|_{H_{0}^{1}(\Omega)} \\
\leq & 2 \epsilon C_{\beta}\|u\|_{\left.\beta^{\alpha / 2, \beta / 2}(I \times \Omega)\right)}\|v\|_{H_{0}^{1}(I \times \Omega)}+N\|v\|_{H_{0}^{1}(\Omega)} \\
\leq & 2 \epsilon C_{\beta}\|u\|_{\left.\beta^{\alpha / 2, \beta / 2}(I \times \Omega)\right)}\|v\|_{H_{0}^{1}(\Omega)}+N\|v\|_{H_{0}^{1}(\Omega)} \\
\leq & 2 \epsilon C_{\beta}\|\phi(x)\|_{L^{2}(U)}\left\|t^{-\alpha}\right\|_{L^{q}(U)}+N \\
\leq & M_{2},
\end{aligned}
$$

where $q=\frac{2}{\alpha}$, and $M_{2}$ is a positive constant.

Thus,

$$
\begin{aligned}
\|\Phi(u)\|= & \sup _{\|v\|_{H_{0}^{1} \leq 1}}|\langle\Phi(u), v\rangle| \\
= & \sup _{\|v\|_{H_{0}^{1} \leq 1}}\left|\langle\phi(u), v\rangle+\langle\psi(x), v\rangle t+\frac{1}{\Gamma(\alpha)} \int_{0}^{t}(t-s)^{\alpha-1}\langle F(u), v\rangle d s\right| \\
\leq & \|\phi(x)\|_{L^{\infty}(\Omega)}\|v\|_{H_{0}^{1}(\Omega)}+\|\psi(x)\|_{L^{\infty}(\Omega)}\|v\|_{H_{0}^{1}(\Omega)} T \\
& +|\langle F(u), v\rangle|\left|\frac{1}{\Gamma(\alpha)} \int_{0}^{t}(t-s)^{\alpha-1} d s\right| \\
\leq & \|\phi(x)\|_{L^{\infty}(\Omega)}+\|\psi(x)\|_{L^{\infty}(\Omega)} T+\left|\frac{M_{2}}{\Gamma(\alpha)} \int_{0}^{t}(t-s)^{\alpha-1} d s\right| \\
\leq & \|\phi(x)\|_{L^{\infty}(\Omega)}+\|\psi(x)\|_{L^{\infty}(\Omega)} T+\frac{M_{2}}{2 \Gamma(\alpha)} t^{\alpha} \\
\leq & \|\phi(x)\|_{L^{\infty}(\Omega)}+\|\psi(x)\|_{L^{\infty}(\Omega)} T+\frac{M_{2}}{2 \Gamma(\alpha)} T^{\alpha} .
\end{aligned}
$$

Hence, $\Phi(u)$ is bounded.

On the other hand, given $\epsilon>0$, set

$$
\delta=\min \left\{\frac{\epsilon}{4\|\psi(x)\|_{L^{\infty}(\mid \Omega)}}, \frac{1}{2}\left(\frac{\epsilon \Gamma(\alpha)}{2 M_{2}}\right)^{\frac{1}{\alpha}}\right\}
$$


Then, for every $v \in H_{0}^{1}(\Omega)$ and all $t_{1}, t_{2} \in[0, T]$ such that $0<t_{2}-t_{1}<\delta$, we obtain

$$
\left\|\Phi u\left(t_{2}\right)-\Phi u\left(t_{1}\right)\right\|_{H^{-1}}=\sup _{\|v\|_{H_{0}^{1}} \leq 1}\left|\left\langle\Phi u\left(t_{2}\right)-\Phi u\left(t_{1}\right), v\right\rangle\right| \leq \epsilon,
$$

that is, $\Phi(u)$ is equicontinuous. In fact,

$$
\begin{aligned}
\left\|\Phi u\left(t_{2}\right)-\Phi u\left(t_{1}\right)\right\|_{H^{-1}}= & \sup _{\|v\|_{H_{0}^{1}} \leq 1}\left|\left\langle\Phi u\left(t_{2}\right)-\Phi u\left(t_{1}\right), v\right\rangle\right| \\
= & \sup _{\|v\|_{H_{0}^{1}} \leq 1} \mid\langle\psi(x), v\rangle\left(t_{2}-t_{1}\right)+\frac{1}{\Gamma(\alpha)} \int_{0}^{t_{2}}\left(t_{2}-s\right)^{\alpha-1}\langle F(u), v\rangle d s \\
& -\frac{1}{\Gamma(\alpha)} \int_{0}^{t_{1}}\left(t_{1}-s\right)^{\alpha-1}\langle F(u), v\rangle d s \mid \\
\leq & \|\psi(x)\|_{L^{\infty}(\Omega)}\|v\|_{H_{0}^{1}}\left|t_{2}-t_{1}\right|+|\langle F(u), v\rangle|\left|\frac{1}{\Gamma(\alpha)} \int_{t_{1}}^{t_{2}}\left(t_{2}-s\right)^{\alpha-1} d s\right| \\
& +|\langle F(u), v\rangle|\left|\frac{1}{\Gamma(\alpha)} \int_{0}^{t_{1}}\left(\left(t_{2}-s\right)^{\alpha-1}-\left(t_{1}-s\right)^{\alpha-1}\right) d s\right| \\
\leq & \|\psi(x)\|_{L^{\infty}(\Omega)}\left|t_{2}-t_{1}\right|+\frac{M_{2}}{\Gamma(\alpha)} \int_{t_{1}}^{t_{2}}\left|\left(t_{2}-s\right)^{\alpha-1}\right| d s \\
& +\frac{M_{2}}{\Gamma(\alpha)} \int_{0}^{t_{1}}\left|\left(t_{2}-s\right)^{\alpha-1}-\left(t_{1}-s\right)^{\alpha-1}\right| d s \\
\leq & \|\psi(x)\|_{L^{\infty}(\Omega)}\left|t_{2}-t_{1}\right|+\frac{M_{2}}{\alpha \Gamma(\alpha)} t_{2}^{\alpha}-\frac{M_{2}}{\alpha \Gamma(\alpha)} t_{1}^{\alpha} \\
\leq & \|\psi(x)\|_{L^{\infty}(\Omega)}\left|t_{2}-t_{1}\right|+\frac{M_{2}}{\alpha \Gamma(\alpha)}\left(t_{2}^{\alpha}-t_{1}^{\alpha}\right) .
\end{aligned}
$$

In the following, we divide the proof into two cases.

Case 1: $\delta \leq t_{1}<t_{2}<T$. Since $1<\alpha<2$, we get

$$
\begin{aligned}
\left\|\Phi u\left(t_{2}\right)-\Phi u\left(t_{1}\right)\right\|_{H^{-1}} & =\sup _{\|v\|_{H_{0}^{1}} \leq 1}\left|\left\langle\Phi u\left(t_{2}\right)-\Phi u\left(t_{1}\right), v\right\rangle\right| \\
& \leq\|\psi(x)\|_{L^{\infty}(\Omega)}\left|t_{2}-t_{1}\right|+\frac{M_{2}}{\alpha \Gamma(\alpha)}\left(t_{2}^{\alpha}-t_{1}^{\alpha}\right) \\
& =\|\psi(x)\|_{L^{\infty}(\Omega)}\left|t_{2}-t_{1}\right|+\frac{M_{2}}{\alpha \Gamma(\alpha)} \alpha t^{\alpha-1}\left(t_{2}-t_{1}\right) \\
& \leq\|\psi(x)\|_{L^{\infty}(\Omega)}\left|t_{2}-t_{1}\right|+\frac{M_{2}}{\Gamma(\alpha)} \delta^{\alpha} \\
& <\|\psi(x)\|_{L^{\infty}(\Omega)} \delta+\frac{M_{2}}{\Gamma(\alpha)} \delta^{\alpha} \\
& <\frac{1}{2} \times \frac{\epsilon}{2}+\left(\frac{1}{2}\right)^{\alpha} \frac{\epsilon}{2} \\
& <\frac{\epsilon}{2}+\frac{\epsilon}{2}=\epsilon .
\end{aligned}
$$

Here $t_{1}<t<t_{2}$, and we applied the mean theorem $t_{2}^{\alpha}-t_{1}^{\alpha}=\alpha t^{\alpha-1}\left(t_{2}-t_{1}\right)$. 
Case 2: $0 \leq t_{1}<\delta, t_{2}<2 \delta$. We have

$$
\begin{aligned}
\left\|\Phi u\left(t_{2}\right)-\Phi u\left(t_{1}\right)\right\|_{H^{-1}} & =\sup _{\|v\|_{H_{0}^{1}} \leq 1}\left|\left\langle\Phi u\left(t_{2}\right)-\Phi u\left(t_{1}\right), v\right\rangle\right| \\
& \leq\|\psi(x)\|_{L^{\infty}(\Omega)}\left|t_{2}-t_{1}\right|+\frac{M_{2}}{\alpha \Gamma(\alpha)}\left(t_{2}^{\alpha}-t_{1}^{\alpha}\right) \\
& \leq\|\psi(x)\|_{L^{\infty}(\Omega)} \delta+\frac{M_{2}}{\alpha \Gamma(\alpha)} t_{2}^{\alpha} \\
& \leq\|\psi(x)\|_{L^{\infty}(\Omega)} \delta+\frac{M_{2}}{\Gamma(\alpha+1)}(2 \delta)^{\alpha} \\
& <\frac{\epsilon}{2}+\frac{\epsilon}{2}=\epsilon .
\end{aligned}
$$

Therefore, $\Phi$ is equicontinuous and uniformly bounded. By the Arzelà-Ascoli theorem we have that $\Phi$ is compact on the space $B^{\alpha / 2, \beta / 2}(I \times \Omega)$, and so the operator $\Phi(u)$ : $B^{\alpha / 2, \beta / 2}(I \times \Omega) \rightarrow B^{\alpha / 2, \beta / 2}(I \times \Omega)$ is completely continuous. This completes the proof of Theorem 3.8 .

Since $B^{\alpha / 2, \beta / 2}(I \times \Omega) \subset C\left([0, T] ; H_{0}^{1}(\Omega)\right)$, combining the results of Theorem 3.8 , by the Schauder fixed point theorem (Lemma 2.4) we obtain that the time-space fractional nonlinear superdiffusion equation (1.2) has a unique weak solution $u \in C\left([0, T] ; H_{0}^{1}(\Omega)\right)$.

\subsection{Existence and uniqueness of weak solutions for the time-space fractional drift superdiffusion equation}

In this part, we consider the existence and uniqueness of weak solution for the time-space fractional drift superdiffusion equation. Analogously, we need some functional spaces and their properties for analyzing a multidimensional time-space fractional drift superdiffusion equation. By Lemmas 2.2 and 2.3 we redefine the following spaces as follows [15, 16]:

$$
B^{\alpha / 2, \gamma / 2}(I \times \Omega):=H^{\alpha / 2}\left(I, L^{2}(\Omega)\right) \cap L^{2}\left(I, H_{0}^{\gamma / 2}(\Omega)\right), \quad 1<\alpha<2, \gamma \in(0,1],
$$

equipped with the norm

$$
\|v\|_{L^{\alpha / 2, \gamma / 2}}(I \times \Omega):=\left(\|v\|_{H^{\alpha / 2}\left(I, L^{2}(\Omega)\right)}^{2}+\|v\|_{L^{2}\left(I, H_{0}^{\gamma / 2}(\Omega)\right)}^{2}\right)^{\frac{1}{2}}, \quad \forall v \in B^{\alpha / 2, \gamma / 2}(I \times \Omega),
$$

where

$$
H^{\alpha / 2}\left(I, L^{2}(\Omega)\right):=\left\{v:\|v(\cdot, t)\|_{L^{2}(\Omega)} \in H^{\alpha / 2}(I)\right\}
$$

endowed with the norm

$$
\|v\|_{H^{\alpha / 2}\left(I, L^{2}(\Omega)\right)}:=\|\| v(\cdot, t)\left\|_{L^{2}(\Omega)}\right\|_{H^{\alpha / 2}(I)} \cdot
$$

Obviously, $B^{\alpha / 2, \gamma / 2}(I \times \Omega)$ is a closed bounded convex subspace (subset) of the standard Banach space $C\left([0, T] ; H_{0}^{1}(\Omega)\right)$. Similarly, we recall the same arguments of Theorem 3.1 in [15] to obtain the following useful inequalities. 
Theorem 3.9 Assume that $1<\alpha<2,0<\gamma \leq 1$, and $\theta_{t}(x, 0)=0$. Then, system (1.3) has a unique weak solution in the space $B^{\alpha / 2, \gamma / 2}(I \times \Omega)$. Furthermore,

$$
\|\theta\|_{B^{\alpha / 2, \gamma / 2(I \times \Omega)}} \leq\|\theta(x, 0)\|_{L^{2}(\Omega)}\left\|t^{-\alpha}\right\|_{L^{q}(\Omega)} \leq\|\phi(x)\|_{L^{2}(\Omega)}\left\|t^{-\alpha}\right\|_{L^{q}(\Omega)}, \quad q=\frac{2}{\alpha} .
$$

Then by Lemma 3.2 problem (1.3) can be reduced to an equivalent integral equation under the fractional-order integral operator $I^{\alpha}$ as in the following problem:

$$
\left\{\begin{array}{cl}
-\phi(x)-\psi(x) t+\theta(x, t) & \\
=\frac{1}{\Gamma(\alpha)} \int_{0}^{t}(t-s)^{\alpha-1}(-\operatorname{div}(u \cdot \theta(x, s)) & \\
\left.\quad+\epsilon \Delta \theta(x, s)-(-\Delta)^{\gamma} \theta(x, s)\right) d s & \text { in } \Omega_{T} \\
\theta(x, t)=0 & \text { on } \partial \Omega_{T} .
\end{array}\right.
$$

Now, we define

$$
\left\{\begin{aligned}
\Phi(\theta)= & \phi(x)+\psi(x) t & & \\
& +\frac{1}{\Gamma(\alpha)} \int_{0}^{t}(t-s)^{\alpha-1}(-\operatorname{div}(u \cdot \theta(x, s)) & & \\
& \left.+\epsilon \Delta \theta(x, s)-(-\Delta)^{\gamma} \theta(x, s)\right) d s & & \text { in } \Omega_{T} \\
\theta(x, t) & =0 & & \text { on } \partial \Omega_{T} .
\end{aligned}\right.
$$

Definition 3.3 We call $\theta \in C\left([0, T] ; H_{0}^{1}(\Omega)\right)$ a weak solution of the time fractional-order drift superdiffusion equation (1.3) if $\int_{\Omega}(\theta-\Phi(\theta)) v d x=0$ for all $t \in[0, T]$ and every $v \in$ $H_{0}^{1}(\Omega)$, that is,

$$
\begin{aligned}
\int_{\Omega} \theta v d x= & \int_{\Omega}\left[\phi(x)+\psi(x) t+\frac{1}{\Gamma(\alpha)} \int_{0}^{t}(t-s)^{\alpha-1}(-\operatorname{div}(u \cdot \theta(x, s))\right. \\
& \left.\left.+\epsilon \Delta \theta(x, s)-(-\Delta)^{\gamma} \theta(x, s)\right) d s\right] v d x .
\end{aligned}
$$

By the definition of (3.7) we get $B^{\alpha / 2, \gamma / 2}(I \times \Omega) \subset C\left([0, T] ; H_{0}^{1}(\Omega)\right)$, and together with the results of Theorem 3.9, we have the following:

Theorem 3.10 If $1<\alpha<2,0<\gamma \leq 1$, and $\theta_{t}(x, 0)=0$, then the operator $\Phi(\theta): B^{\alpha / 2, \gamma / 2}(I \times$ $\Omega) \rightarrow B^{\alpha / 2, \gamma / 2}(I \times \Omega)$ is completely continuous.

Proof Put

$$
F(\theta)=-\operatorname{div}(u \cdot \theta(x, t))+\epsilon \Delta \theta(x, t)-(-\Delta)^{\gamma} \theta(x, t) .
$$

We can rewrite

$$
\Phi(\theta)=\phi(x)+\psi(x) t+\frac{1}{\Gamma(\alpha)} \int_{0}^{t}(t-s)^{\alpha-1} F(\theta) d s .
$$

For each $v \in H_{0}^{1}(\Omega)$ such that $\|v\|_{H_{0}^{1}(\Omega)} \leq 1$, integrating by parts, in terms of the Riesz fractional derivative in Definition 2.4 and Lemma 3.3, since $0<\gamma<1$, by Lemma 3.4, together with the real-order Sobolev imbedding theorem, we have that $H_{0}^{1}(\Omega) \hookrightarrow H_{0}^{\gamma / 2}(\Omega) \hookrightarrow$ 
$L^{2}(\Omega)$, and thus $\|\theta\|_{L^{2}}(\Omega) \leq\|\theta\|_{H_{0}^{\gamma / 2}(\Omega)} \leq\|\theta\|_{H_{0}^{1}(\Omega)}$. In the following, we denote $\|\theta\|_{H_{0}^{1}(\Omega)}$ and $\|\theta\|_{H_{0}^{-1}(\Omega)}$ by $\|\theta\|_{H_{0}^{1}}$ and $\|\theta\|_{H_{0}^{-1}}$, respectively. Hence, by the Cauchy-Schwarz and Hölder inequalities and by the standard Sobolev imbedding theorem, Theorem 3.9, and Lemma 3.5 , since $1<\alpha<2$, we obtain

$$
\begin{aligned}
|\langle F(\theta), v\rangle|= & \left|\int_{\Omega}(u \cdot \theta+\epsilon \nabla \theta) \cdot \nabla v d x+\int_{\Omega}\left(-(-\Delta)^{\gamma}\right) \theta(x, t) v d x\right| \\
\leq & {\left[\|u\|_{L^{2}(\Omega)}\left(\int_{\Omega}|\theta|^{2} d x\right)^{\frac{1}{2}}+|\epsilon|\left(\int_{\Omega}|\nabla \theta|^{2} d x\right)^{\frac{1}{2}}\right]\left(\int_{\Omega}|\nabla v|^{2} d x\right)^{\frac{1}{2}} } \\
& +\left|\left(\left(-(-\Delta)^{\gamma}\right) \theta(x, t), v(x, t)\right)\right| \\
\leq & \left(\|u\|_{L^{2}(\Omega)}\|\theta\|_{L^{2}(\Omega)}+|\epsilon|\|\nabla \theta\|_{L^{2}(\Omega)}\right)\|\nabla v\|_{L^{2}(\Omega)} \\
& +\left|\left(\frac{\partial D^{2 \gamma}}{\partial|x|} \theta(x, t), v(x, t)\right)\right| \\
\leq & \left(\|u\|_{L^{2}(\Omega)}\|\theta\|_{L^{2}(\Omega)}+|\epsilon|\|\nabla \theta\|_{L^{2}(\Omega)}\right)\|\nabla v\|_{L^{2}(\Omega)} \\
& +\left|-C_{\gamma}\left[\left({ }_{a}^{R} D_{x}^{\gamma} \theta{ }_{x}^{R} D_{b}^{\gamma} v\right)_{L^{2}(U)}+\left({ }_{x}^{R} D_{b}^{\gamma} \theta{ }_{a}^{R} D_{x}^{\gamma} v\right)_{L^{2}(U)}\right]\right| \\
\leq & C_{1}\left(\|u\|_{L^{2}(\Omega)}\|\theta\|_{L^{2}(U)}+|\epsilon|\|\theta\|_{L^{2}(U)}\right)\|v\|_{H_{0}^{1}(\Omega)} \\
& +C_{\gamma}\left[\left\|_{a}^{R} D_{x}^{\gamma} \theta\right\|_{L^{2}(U)}\left\|{ }_{x}^{R} D_{b}^{\gamma} v\right\|_{L^{2}(U)}+\left\|{ }_{x}^{R} D_{b}^{\gamma} \theta\right\|_{L^{2}(U)}\|\|_{a}^{R} D_{x}^{\gamma} v \|_{L^{2}(U)}\right] \\
\leq & C_{1}\left(\|u\|_{L^{2}(\Omega)}+\max |\epsilon|\right)\|\theta\|_{L^{2}\left(I, H^{\gamma / 2}(\Omega)\right)}\|v\|_{H_{0}^{1}(\Omega)} \\
& +2 C_{\gamma}\|\theta\|_{L^{2}(I, H \gamma / 2(\Omega))}\|v\|_{H_{0}^{\gamma / 2}\left(I, L^{2}(\Omega)\right)} \\
= & C_{2}\left(\|u\|_{L^{2}(\Omega)}+\max |\epsilon|\right)\|\theta\|_{B^{\alpha / 2, \gamma / 2}(I \times \Omega)}\|v\|_{H_{0}^{1}(\Omega)} \\
& +2 C_{\gamma}\|\theta\|_{B^{\alpha / 2, \gamma / 2}(I \times \Omega)}\|v\|_{H_{0}^{1}(I \times \Omega)} \\
\leq & C_{3}\left(\|u\|_{L^{2}(\Omega)}+\max |\epsilon|\right)\|\theta\|_{B^{\frac{\alpha}{2}, \frac{\gamma}{2}}(I \times \Omega)}\|v\|_{H_{0}^{1}(\Omega)} \\
& +2 C_{\gamma}\|\theta\|_{B^{\alpha / 2, \gamma / 2}(I \times \Omega)}\|v\|_{H_{0}^{1}(\Omega)} \\
\leq & C_{3}\left(\|u\|_{L^{2}(\Omega)}+\max |\epsilon|\right)\|\phi(x)\|_{L^{2}(\Omega)}\left\|t^{-\alpha}\right\|_{L^{q}(\Omega)} \\
& +2 C_{\gamma}\|\phi(x)\|_{L^{2}(\Omega)}\left\|t^{-\alpha}\right\|_{L^{q}(\Omega)} \\
\leq & C\|\phi\|_{L^{2}(\Omega)}\left\|t^{-\alpha}\right\|_{L^{q}(\Omega)} \leq M_{3} . \\
& \\
& \\
&
\end{aligned}
$$

Here, $C_{1}, C_{2}, C_{3}$ denote the best Sobolev constants, respectively, $C=\max \left\{C_{3}\left(\|u\|_{L^{2}(\Omega)}+\right.\right.$ $\left.\max |\epsilon|)+2 C_{\gamma}\right\}$, and $M_{3}$ is a positive constant.

Thus, by the Cauchy-Schwarz inequality we obtain

$$
\begin{aligned}
\|\Phi(\theta)\|_{H^{-1}} & =\sup _{\|v\|_{H_{0}^{1}} \leq 1}|\langle\Phi(\theta), v\rangle| \\
& =\sup _{\|v\|_{H_{0}^{1}} \leq 1}\left|\langle\phi(x), v\rangle+\langle\psi(x), v\rangle t+\frac{1}{\Gamma(\alpha)} \int_{0}^{t}(t-s)^{\alpha-1}\langle F(\theta), v\rangle d s\right| \\
& \leq|\langle\phi(x), v\rangle|+\mid\left\langle\psi(x), v|t|+\left|\frac{1}{\Gamma(\alpha)} \int_{0}^{t}(t-s)^{\alpha-1}\langle F(\theta), v\rangle d s\right|\right. \\
& \leq\|\phi(x)\|_{L^{\infty}(\Omega)}\|v\|_{H_{0}^{1}}+\|\psi(x)\|_{L^{\infty}(\Omega)}\|v\|_{H_{0}^{1}} T
\end{aligned}
$$




$$
\begin{aligned}
& +|\langle F(\theta), v\rangle|\left|\frac{1}{\Gamma(\alpha)} \int_{0}^{t}(t-s)^{\alpha-1} d s\right| \\
\leq & \|\phi(x)\|_{L^{\infty}(\Omega)}+\|\psi(x)\|_{L^{\infty}(\Omega)} T+\frac{M_{3}}{\Gamma(\alpha)}\left|\int_{0}^{t}(t-s)^{\alpha-1} d s\right| \\
\leq & \|\phi(x)\|_{L^{\infty}(\Omega)}+\|\psi(x)\|_{L^{\infty}(\Omega)} T+\frac{M_{3}}{\alpha \Gamma(\alpha)} t^{\alpha} \\
\leq & \|\phi(x)\|_{L^{\infty}(\Omega)}+\|\psi(x)\|_{L^{\infty}(\Omega)} T+\frac{M_{3}}{\alpha \Gamma(\alpha)} T^{\alpha} .
\end{aligned}
$$

Hence, $\Phi(\theta)$ is bounded.

On the other hand, given $\epsilon>0$, set

$$
\delta=\min \left\{\frac{\epsilon}{4\|\psi(x)\|_{L^{\infty}(\Omega)}}, \frac{1}{2}\left(\frac{\epsilon \Gamma(\alpha)}{2 M_{3}}\right)^{\frac{1}{\alpha}}\right\} .
$$

Then, for every $v \in H_{0}^{1}(\Omega)$ and all $t_{1}, t_{2} \in[0, T]$ such that $0<t_{2}-t_{1}<\delta$, we have

$$
\left\|\Phi \theta\left(t_{2}\right)-\Phi \theta\left(t_{1}\right)\right\|_{H^{-1}}=\sup _{\|v\|_{H_{0}^{1}} \leq 1}\left|\left\langle\Phi \theta\left(t_{2}\right)-\Phi \theta\left(t_{1}\right), v\right\rangle\right| \leq \epsilon,
$$

that is, $\Phi(\theta)$ is equicontinuous. In fact,

$$
\begin{aligned}
\left\|\Phi \theta\left(t_{2}\right)-\Phi \theta\left(t_{1}\right)\right\|_{H^{-1}}= & \sup _{\|\nu\|_{H_{0}^{1}} \leq 1}\left|\left\langle\Phi \theta\left(t_{2}\right)-\Phi \theta\left(t_{1}\right), v\right\rangle\right| \\
= & \sup _{\|\nu\|_{H_{0}^{1}} \leq 1} \mid\langle\psi(x), v\rangle\left(t_{2}-t_{1}\right)+\frac{1}{\Gamma(\alpha)} \int_{0}^{t_{2}}\left(t_{2}-s\right)^{\alpha-1}\langle F(\theta), v\rangle d s \\
& -\frac{1}{\Gamma(\alpha)} \int_{0}^{t_{1}}\left(t_{1}-s\right)^{\alpha-1}\langle F(\theta), v\rangle d s \mid \\
\leq & \|\psi(x)\|_{L^{\infty}(\Omega)}\|v\|_{H_{0}^{1}}\left|t_{2}-t_{1}\right| \\
& +|\langle F(\theta), v\rangle|\left|\frac{1}{\Gamma(\alpha)} \int_{t_{1}}^{t_{2}}\left(t_{2}-s\right)^{\alpha-1} d s\right| \\
& +|\langle F(\theta), v\rangle|\left|\frac{1}{\Gamma(\alpha)} \int_{0}^{t_{1}}\left(\left(t_{2}-s\right)^{\alpha-1}-\left(t_{1}-s\right)^{\alpha-1}\right) d s\right| \\
\leq & \|\psi(x)\|_{L^{\infty}(\Omega)}\left|t_{2}-t_{1}\right|+\frac{M_{3}}{\Gamma(\alpha)} \int_{t_{1}}^{t_{2}}\left|\left(t_{2}-s\right)^{\alpha-1}\right| d s \\
& +\frac{M_{3}}{\Gamma(\alpha)} \int_{0}^{t_{1}}\left|\left(t_{2}-s\right)^{\alpha-1}-\left(t_{1}-s\right)^{\alpha-1}\right| d s \\
\leq & \|\psi(x)\|_{L^{\infty}(\Omega)}\left|t_{2}-t_{1}\right|+\frac{M_{3}}{\alpha \Gamma(\alpha)} t_{2}^{\alpha}-\frac{M_{3}}{\alpha \Gamma(\alpha)} t_{1}^{\alpha} \\
\leq & \|\psi(x)\|_{L^{\infty}(\Omega)}\left|t_{2}-t_{1}\right|+\frac{M_{3}}{\alpha \Gamma(\alpha)}\left(t_{2}^{\alpha}-t_{1}^{\alpha}\right) .
\end{aligned}
$$

In the following, we divide the proof into two cases. 
Case 1: $\delta \leq t_{1}<t_{2}<T$. Since $1<\alpha<2$, we get

$$
\begin{aligned}
\left\|\Phi \theta\left(t_{2}\right)-\Phi \theta\left(t_{1}\right)\right\|_{H^{-1}} & =\sup _{\|\nu\|_{H_{0}^{1}} \leq 1}\left|\left\langle\Phi \theta\left(t_{2}\right)-\Phi \theta\left(t_{1}\right), v\right\rangle\right| \\
& \leq\|\psi(x)\|_{L^{\infty}(\Omega)}\left|t_{2}-t_{1}\right|+\frac{M_{3}}{\alpha \Gamma(\alpha)}\left(t_{2}^{\alpha}-t_{1}^{\alpha}\right) \\
& =\|\psi(x)\|_{L^{\infty}(\Omega)}\left|t_{2}-t_{1}\right|+\frac{M_{3}}{\alpha \Gamma(\alpha)} \alpha t^{\alpha-1}\left(t_{2}-t_{1}\right) \\
& \leq\|\psi(x)\|_{L^{\infty}(\Omega)}\left|t_{2}-t_{1}\right|+\frac{M_{3}}{\Gamma(\alpha)} \delta^{\alpha} \\
& <\|\psi(x)\|_{L^{\infty}(\Omega)} \delta+\frac{M_{3}}{\Gamma(\alpha)} \delta^{\alpha} \\
& <\frac{1}{2} \times \frac{\epsilon}{2}+\left(\frac{1}{2}\right)^{\alpha} \frac{\epsilon}{2}<\frac{\epsilon}{2}+\frac{\epsilon}{2}=\epsilon .
\end{aligned}
$$

Here $t_{1}<t<t_{2}$, and we applied the mean theorem $t_{2}^{\alpha}-t_{1}^{\alpha}=\alpha t^{\alpha-1}\left(t_{2}-t_{1}\right)$.

Case 2: $0 \leq t_{1}<\delta, t_{2}<2 \delta$. We have

$$
\begin{aligned}
\left\|\Phi \theta\left(t_{2}\right)-\Phi \theta\left(t_{1}\right)\right\|_{H^{-1}} & \left.=\sup _{\|v\|_{H_{0}^{1}} \leq 1}|| \Phi \theta\left(t_{2}\right)-\Phi \theta\left(t_{1}\right), v\right\rangle \mid \\
& \leq\|\psi(x)\|_{L^{\infty}(\Omega)}\left|t_{2}-t_{1}\right|+\frac{M_{3}}{\alpha \Gamma(\alpha)}\left(t_{2}^{\alpha}-t_{1}^{\alpha}\right) \\
& \leq\|\psi(x)\|_{L^{\infty}(\Omega)} \delta+\frac{M_{3}}{\alpha \Gamma(\alpha)} t_{2}^{\alpha} \\
& \leq\|\psi(x)\|_{L^{\infty}(\Omega)} \delta+\frac{M_{3}}{\Gamma(\alpha+1)}(2 \delta)^{\alpha} \\
& <\frac{\epsilon}{2}+\frac{\epsilon}{2}=\epsilon .
\end{aligned}
$$

Therefore, $\Phi$ is equicontinuous and uniformly bounded. By the Arzelà-Ascoli compactness theorem, $\Phi$ is compact on the space $B^{\alpha / 2, \gamma / 2}(I \times \Omega)$, and thus the operator $\Phi(u): B^{\alpha / 2, \gamma / 2}(I \times \Omega) \rightarrow B^{\alpha / 2, \gamma / 2}(I \times \Omega)$ is completely continuous. This completes the proof of Theorem 3.10.

Since $B^{\alpha / 2, \gamma / 2}(I \times \Omega) \subset C\left([0, T] ; H_{0}^{1}(\Omega)\right)$, by Theorem 3.10, using the Schauder fixed point theorem (Lemma 2.4), we obtain that the multidimensional fractional drift superdiffusion equation (1.3) has a unique weak solution $\theta \in C\left([0, T] ; H_{0}^{1}(\Omega)\right)$.

\section{The conclusions}

In this paper, we presented the existence and uniqueness of weak solutions in a standard Banach space $C\left([0, T] ; H_{0}^{1}(\Omega)\right)$ for a class of fractional superdiffusion initial-boundary value problems. For a multidimensional time fractional drift superdiffusion equation, we only considered the simplest case, which includes an artificial diffusion term and assume that the smooth divergence-free vector field (i.e., the drift velocity) $\mathbf{u} \in L^{2}(\Omega)$ only depends on the spatial variable $x$. Then, by recalling the arguments in Theorem 3.1 in [15] we obtained two important inequalities (2.18) and (3.10). Finally, by exploiting the Schauder fixed point theorem and the Arzelà-Ascoli compactness theorem we obtained the unique 
weak solutions for the time-space fractional superdiffusion equation (1.1), the time-space fractional nonlinear superdiffusion equation (1.2), and the multidimensional fractional drift superdiffusion equation (1.3).

\section{Competing interests}

The authors declare that they have no competing interests.

\section{Authors' contributions}

All authors contributed equally to the writing of this paper. All authors read and approved the final manuscript.

\section{Author details}

${ }^{1}$ School of Mathematics and Statistics, Xi'an Jiaotong University, Xi'an, 710049, China. ${ }^{2}$ Department of Mathematics, Yunnan Nationalities University, Kunming, 650031, China. ${ }^{3}$ Institute of Mathematics, Yunnan Normal University, Kunming, 650092, China.

\section{Acknowledgements}

The work is supported by National Natural Science Foundation of China (Nos. 11371289, 11501441, 11161057, 11561076, 11426068) and Talent Introduction Fund Projects (2015JB018).

Received: 18 September 2016 Accepted: 2 December 2016 Published online: 02 January 2017

\section{References}

1. Zola, RS, Lenzi, EK, Evangelista, LR, et al.: Exact solutions for a diffusion equation with a nonlinear external force. Phys. Lett. A 372, 2359-2363 (2008)

2. Liang, J, Ren, F, Qiu, W, et al.: Exact solutions for nonlinear fractional anomalous diffusion equations. Physica A 385 80-94 (2007)

3. Ma, J, Liu, Y: Exact solutions for a generalized nonlinear fractional Fokker-Planck equation. Nonlinear Anal. 11, 515-521 (2010)

4. GaLychuk, W, Datsko, BY: Pattern formation in a fractional reaction diffusion system. Physica A 365(2), $300-306$ (2006)

5. Hilfer, R: Applications of Fractional Calculus in Physics. World Scientific, Singapore (2000)

6. Marin, M: On existence and uniqueness in thermoelasticity of micropolar bodies. C. R. Math. Acad. Sci. Paris 321(12), 475-480 (1995)

7. Marin, M: Some basic theorems in elastostatics of micropolar materials with voids. J. Comput. Appl. Math. 70(1), 115-126 (1996)

8. Marin, M, Marinescu, C: Thermoelasticity of initially stressed bodies. Asymptotic equipartition of energies. Int. J. Eng. Sci. 36(1), 73-86 (1998)

9. Zhang, Y, Benson, DA, Reeves, DM: Time and space nonlocalities underlying fractional-derivative models: distinction and literature review of field applications. Adv. Water Resour. 32, 561-581 (2009)

10. Podlubny, I: Fractional Differential Equations. Academic Press, New York (1999)

11. Kilbas, A, Srivastava, HM, Trujillo, JJ: Theory and Application of Fractional Differential Equations. Elsevier, Amsterdam (2006)

12. Mainardi, F, Luchko, Y, Pagnini, G: The fundamental solution of the space-time fractional diffusion equation. Fract. Calc. Appl. Anal. 4, 153-192 (2001)

13. Liu, FW, Anh, V, Turner, l, et al.: Time fractional advection-dispersion equation. J. Appl. Math. Comput. 13, 233-246 (2003)

14. Gorenflo, R, Luchko, Y, Mainardi, F: Wright function as scale-invariant solutions of diffusion-wave equation. J. Comput. Appl. Math. 118, 175-191 (2000)

15. $\mathrm{Li}, \mathrm{XJ}, \mathrm{Xu}, \mathrm{CJ}$ : Existence and uniqueness of the weak solution of the space-time fractional diffusion equation and a spectral method approximation. Commun. Comput. Phys. 8, 1016-1051 (2010)

16. Zhao, J, Xiao, JY, Xu, Y: A finite element method for the multiterm time-space Riesz fractional advection-diffusion equations in finite domain. Abstr. Appl. Anal. 2013, Article ID 868035 (2013)

17. Fan, QY, Wang, WT, Yi, XJ: Anti-periodic solutions for a class of nonlinear nth-order differential equations with delays. J. Comput. Appl. Math. 230(2), 762-769 (2009)

18. Wang, WB, Shen, JH: Existence of solutions for anti-periodic boundary value problems. Nonlinear Anal. 70(2), 598-605 (2009)

19. Baleanu, D, Uğurlu, E: Regular fractional dissipative boundary value problems. Adv. Differ. Equ. 2016, Article ID 175 (2016). doi:10.1186/s13662-016-0883-6

20. Chen, AP, Chen, Y: Existence of solutions to anti-periodic boundary value problem for nonlinear fractional differential equations. Differ. Equ. Dyn. Syst. 19(3), 237-252 (2011)

21. Chen, AP, Tian, Y: Existence of three positive solutions to three-point boundary value problem of nonlinear fractional differential equation. Differ. Equ. Dyn. Syst. 18(3), 327-339 (2010)

22. Ahmad, B: Existence of solutions for fractional differential equations of order $q \in(2,3]$ with anti-periodic boundary conditions. J. Appl. Math. Comput. 34, 385-391 (2010)

23. Bai, ZB, Lü, H: Positive solutions for boundary value problem of nonlinear fractional differential equation. J. Math. Anal. Appl. 311, 495-505 (2005)

24. Zhang, SS: Positive solutions for boundary value problem of nonlinear fractional differential equations. Electron. J. Differ. Equ. 2006, 36 (2006)

25. Zhang, SS: Existence of positive solution for some class of nonlinear fractional differential equations. J. Math. Anal. Appl. 278, 136-148 (2003) 
26. Qiu, ML, Mei, LQ: Existence of weak solutions for nonlinear time-fractional $p$-Laplace problems. J. Appl. Math. 2014, Article ID 231892 (2014)

27. Qiu, ML, Mei, LQ, Yang, GS, Yuan, XZ: Solutions for $p$-Laplace problems with nonlinear time-fractional differential equation. J. Inequal. Appl. 2014, Article ID 262 (2014)

28. Gorenflo, R, Mainardi, F: Random walk models for space-fractional diffusion processes. Fract. Calc. Appl. Anal. 1(2), 167-191 (1998)

29. Yang, Q, Liu, FW, Turner, I: Numerical methods for fractional partial differential equations with Riesz space fractional derivatives. Appl. Math. Model. 34, 200-218 (2010)

30. Wu, GC, Baleanu, D, Xie, HP: Riesz Riemann-Liouville difference on discrete domains. Chaos 26, 084308 (2016)

31. Klafter, J, White, B, Levandowsky, M: Microzooplankton feeding behavior and the Lévy walk. In: Biological Motion. Lecture Notes in Biomathematics, pp. 281-293. Springer, Berlin (1990)

32. Caffarelli, LA, Vasseur, A: Drift diffusion equations with fractional diffusion and the quasi-geostrophic equation. Ann. Math. 171, 1903-1930 (2010)

33. Wu, GC, Baleanu, D, Deng, ZG, Zeng, SD: Lattice fractional diffusion equation in terms of a Riesz-Caputo difference. Physica A 438, 335-339 (2015)

34. El-Sayed, AMA, Gaber, M: On the finite Caputo and finite Riesz derivatives. Electron. J. Theor. Phys. 3, 81-95 (2006)

35. Muslih, Sl, Agrawal, OP: Riesz fractional derivatives and fractional dimensional space. Int. J. Theor. Phys. 49, 270-275 (2010)

36. Adams, RA, John, JFF: Sobolev Spaces. Academic Press New York (2009)

37. Li, KT, Ma, YC, Wang, LZ: The Generalized Function and Sobolev Space. Xi'an Jiaotong University Press, Shaanxi (2008)

38. Corduneanu, C: Integral Equations and Applications. Cambridge University Press, New York (1973)

39. Ben Amar, A, Jeribi, A, Mnif, M: Some fixed point theorems and application to biological model. Numer. Funct. Anal. Optim. 29, 1-23 (2008)

40. Barroso, CS: Krasnosel'skij fixed point theorem for weakly continuous maps. Nonlinear Anal., Theory Methods Appl. 55, 25-31 (2003)

41. Ben Amar, A, Jeribi, A, Mnif, M: On a generalization of the Schauder and Krasnosel'skii fixed point theorems on Dunford-Pettis spaces and applications. Math. Methods Appl. Sci. 28, 1737-1756 (2005)

42. Latrach, K, Taoudi, MA, Zeghal, A: Some fixed point theorems of the Schauder and the Krasnosel'skii type and application to nonlinear transport equations. J. Differ. Equ. 221, 256-271 (2006)

43. Zhang, SS, Yang, GS: Some further generalizations of Ky Fan's minimax inequality and its applications to variational inequalities. Appl. Math. Mech. 11(11), 1027-1034 (1990)

\section{Submit your manuscript to a SpringerOpen ${ }^{\circ}$ journal and benefit from:}

- Convenient online submission

Rigorous peer review

- Immediate publication on acceptance

- Open access: articles freely available online

- High visibility within the field

- Retaining the copyright to your article 